\title{
Kinetics observations of bacterial cellulose thickness formation using image processing approach during the fermentation process
}

\author{
${ }^{1}$ Nugroho, D.A., ${ }^{2, *}$ Sutiarso, L., ${ }^{3}$ Rahayu, E.S. and ${ }^{2}$ Masithoh, R.E. \\ ${ }^{1}$ Department of Agroindustrial Technology, Faculty of Agricultural Technology, Universitas Gadjah \\ Mada,Jl. Flora No. 1, Bulaksumur, Yogyakarta 55281, Indonesia \\ ${ }^{2}$ Department of Agricultural and Biosystems Engineering, Faculty of Agricultural Technology, Universitas \\ Gadjah Mada, Jl. Flora No. 1, Bulaksumur, Yogyakarta 55281, Indonesia \\ ${ }^{3}$ Department of Food and Agricultural Products Technology, Faculty of Agricultural \\ Technology,Universitas Gadjah Mada, Jl. Flora No. 1, Bulaksumur, Yogyakarta 55281, Indonesia
}

\begin{abstract}
Article history:
Received: 5 February 2021

Received in revised form: 26 March 2021

Accepted: 10 June 2021

Available Online: 9 February 2022
\end{abstract}

\section{Keywords:}

Bacterial cellulose,

$\mathrm{BC}$ sheet,

Cluster,

Gompertz model

DOI:

https://doi.org/10.26656/fr.2017.6(1).093

\begin{abstract}
Bacterial cellulose is an exopolysaccharide that has a higher level of purity compared to cellulose from plants. Bacterial Cellulose (BC) is widely used for various uses so that it requires certain initial conditions, one of which is thickness. During the fermentation process, cellulose will be secreted into the medium to form BC sheets and visually visible over the time period. The aim of this research was to study the relationship between variables that influence during the fermentation and to fit the kinetic model of the $\mathrm{BC}$ thickness formation using image processing approach during the fermentation process. A USB camera was placed in front of the fermenter to capture the formation of $\mathrm{BC}$ thickness. Python programming language was used to process the image and calculated the thickness of the $\mathrm{BC}$ sheet from the beginning to the end of the fermentation process. Several supporting parameters were observed by placing the turbidity, $\mathrm{pH}$, and medium temperature sensors. Observations were made in real time with a range of data collection every 15 mins during fermentation. The highest correlation value was obtained from the relationship between time and thickness. The fermentation process is divided into 2 clusters, a change in cluster occurs at the 61 st hour. The model that describes the relationship between time and thickness was the Gompertz model.
\end{abstract}

\section{Introduction}

Bacterial Cellulose (BC) is an exopolysaccharide that has a higher level of purity compared to plant cellulose, produced by several types of microorganisms (Chawla et al., 2009). It is a biopolymer with many purposes of uses. It has a better material property, including high purity, high degree of porosity, relatively high permeability to liquid and gases, high water-uptake capacity, tensile strength, and ultrafine network (Ullah et al., 2016). BC is widely used for purposes in various fields. In the food sector, it is widely used as a dessert drink (Iguchi et al., 2000), a low cholesterol diet (Chau et al., 2008), a meat substitute for vegetarians with the addition of Monascus purpureus (Purwadaria et al., 2010), as well as a food additive as an ingredient for enzyme immobilization ( $\mathrm{Wu}$ and Lia, 2008).

The use of $\mathrm{BC}$ material for various applications requires different initial conditions for $\mathrm{BC}$ material, such as shape, size, and thickness. BC membranes of various thicknesses ranging from $5 \mathrm{~mm}, 7 \mathrm{~mm}$ and $9 \mathrm{~mm}$ were used as guided bone regeneration (Lee et al., 2017). In vitro drug release rate was dependent on the $\mathrm{BC}$ film thickness (Maver et al., 2015). Modification of the fermentation process was carried out to obtain BC with a thickness of up to $30 \mathrm{~mm}$ compared to conventional methods (Hsieh et al., 2016). For this reason, this study aims to monitor the rate of $\mathrm{BC}$ thickness formation using an image processing approach during the fermentation process.

The high yield cellulose production capacity is the most crucial parameter in $\mathrm{BC}$ production during the fermentation process (Bilgi et al., 2016). The increase of growth time will increase the formation of $\mathrm{BC}$ along with hydrogen and $\mathrm{C}-\mathrm{H}$ bonding (Sheykhnazari et al., 2011). The growth of $B C$ formation can be seen by the thickness over the time of fermentation passes (Nugroho et al., 2020). Several conditions that influenced the 
formation of $\mathrm{BC}$ were fermentation time (Iguchi et al., 2000), temperature (Mohammad and Rahman, 2014), turbidity, $\mathrm{pH}$, and thickness of BC (Hsieh et al., 2016).

The method of observing the changes in $\mathrm{BC}$ thickness in real time using image processing has been reported (Nugroho et al., 2020). The thickness measurement algorithm has succeeded in separating BC sheet objects from other objects such as medium, nonsheet $\mathrm{BC}$ and air space between the medium and the fermenter cover (Figure 1). This research is aimed to study the relationship between variables that influence during the fermentation and to fit a kinetic model of the $\mathrm{BC}$ thickness formation using image processing approach during the fermentation process.

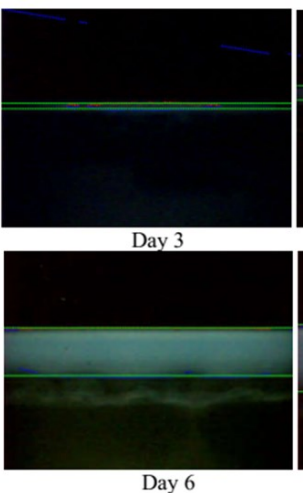

Day 6

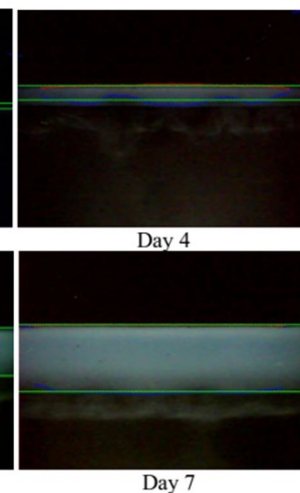

Day

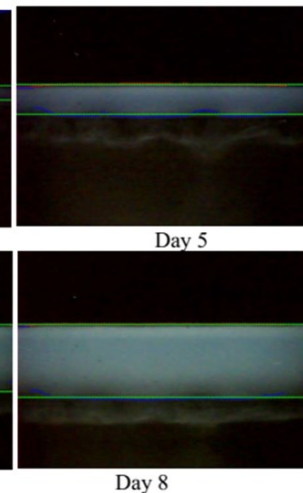

Day 8
Figure 1. BC sheet thickness measured using image processing during fermentation at room temperature without agitating and stirring.

\section{Materials and methods}

\subsection{Materials}

The composition medium for this fermentation were consisted of $1.5 \mathrm{~L}$ coconut water, $5 \%$ sucrose, $1.5 \%$ ammonium sulphate, and acetic acid for adjusting $\mathrm{pH}$ to 4.0 (Nugroho et al., 2020). The medium was sterilized for 15 mins and then allowed to cool. The fermentation process began by pouring the medium into the fermenter followed by inoculating $5 \%$ of the stock culture of Gluconacetobacter xylinus into the fermenter.

\subsection{Fermenter}

The fermenter was built using acrylic material that is given a non-reflective type of black paint on the outer surface with a length of $35 \mathrm{~cm}$, a width of $25 \mathrm{~cm}$ and a height of $6 \mathrm{~cm}$, and provides a transparent field facing the USB camera of $6 \mathrm{~cm}$ width and $4 \mathrm{~cm}$ height. A 145 lux LED lamp (measured with luxmeter LX-101) was positioned in front of the fermenter to provide lighting assistance and increase object contrast. An HD type USB camera used with the brand name Alloet (maximum resolution of $1280 \times 960$ pixels, CMOS sensor type, and autofocus) is placed right in front of the transparent field (Figure 2). The fermenter was operated without stirring and agitating, at room temperature, in aerobic condition. The dimension of the fermenter gives a wide surface area for contact with the oxygen and the fermenter lid was also made from paper which oxygen could pass through.

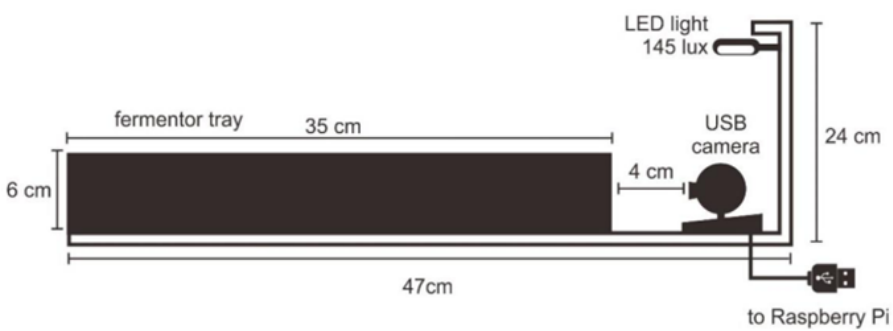

Figure 2. Arrangement of fermenter for image processing purpose (Nugroho et al., 2020).

\subsection{Equipment used}

The sensor equipment was placed through the fermenter cover, which consists of DS18B20 Temperature Sensor Module Kit Waterproof with Digital Sensor Cable and Stainless-Steel Probe Terminal Adapter for Arduino, Keyestudio Turbidity Sensor V1.0 Compatible with Arduino and PH4502C pH Meter Value Detector Module Detection with Module Monitoring Controller and BNC Block Electrode Probe. All the sensors were placed submerged in the fermentation medium $1 \mathrm{~cm}$ from the bottom of the fermenter. Those sensors were connected to the Arduino Uno. The Arduino Uno is a microcontroller board based on the ATmega328. It has six analog inputs, a $16-\mathrm{MHz}$ crystal oscillator, a USB connection, a power jack, and an InCircuit Serial Programming header (D'Ausilio, 2012). The sensors connected to Arduino Uno will provide current reading information, which will be converted into digital data that can be read by the Raspberry Pi 3. In contrast, the USB Camera is directly connected to the Raspberry Pi.

Raspberry Pi 3 was an economical, palm-sized computer. This board had 4x USB ports onboard, an Ethernet port, Wi-Fi capability, HDMI port, SD card slot, and 40 pins. It was installed with Raspbian OS, a free and open-source software. The OS was based on the Linux kernel. The BC thickness calculation algorithm and command insertion method into the MySQL database (Nugroho et al., 2020) was developed in python language programming.

\subsection{Data collection}

Data collection of reading sensor values was done by sending the values from Arduino to Raspberry Pi 3, while data from the USB camera was directly sent to Raspberry Pi 3 through the $\mathrm{BC}$ thickness calculation method first (Figure 3). All the data were inputted into MySQL database that is also already installed on Raspberry Pi 3. Data was taken every 15 mins from the 
beginning of fermentation until 8 days.

The reading data is stored in the MySQL database every $15 \mathrm{mins}$, and at the same time, the data was sent to the user's email using the http server, which was also installed on the Raspberry Pi 3 via internet access.

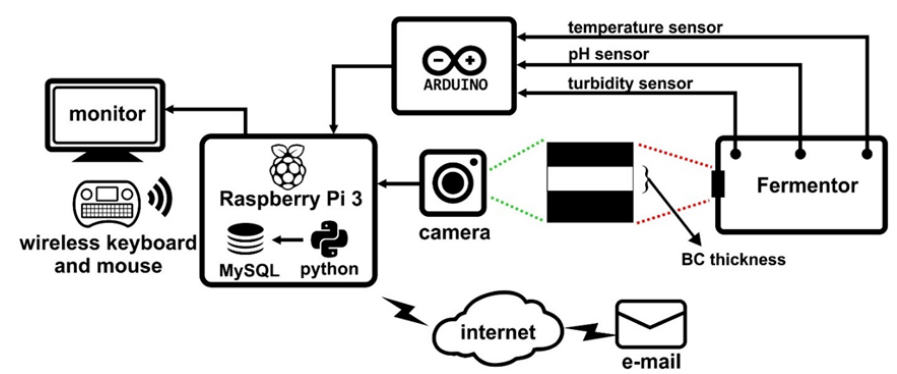

Figure 3. Dataflow process of $\mathrm{BC}$ fermentation monitoring

Data obtained during fermentation was then analyzed using the $\mathrm{R}$ programming language for timeperiod data reading, PCA analysis, and kinetic analysis. The $\mathrm{R}$ version is 3.6.3, downloaded from https://www.rproject.org, while the GUI application uses RStudio Desktop version 1.3.1093, downloaded from https:// download1.rstudio.org/desktop/windows/RStudio1.3.1093.exe.

\section{Results and discussion}

During the fermentation process, the four sensors installed on the fermenter, namely the temperature sensor, $\mathrm{pH}$ sensor, turbidity sensor and camera, provide data readings which were plotted in a graph as shown in Figure 4. During the fermentation process, the newly inoculated microorganisms need the amount of time for adaptation, increase the amount of biomass, and produce metabolites. However, because the amount of biomass is still small, the metabolite results in the form of $\mathrm{BC}$ sheets are not visually visible nor thickness figures can be read. The rate of increase in thickness of the BC sheet begins after 61 hours and continues to increase significantly until the end of the fermentation process.

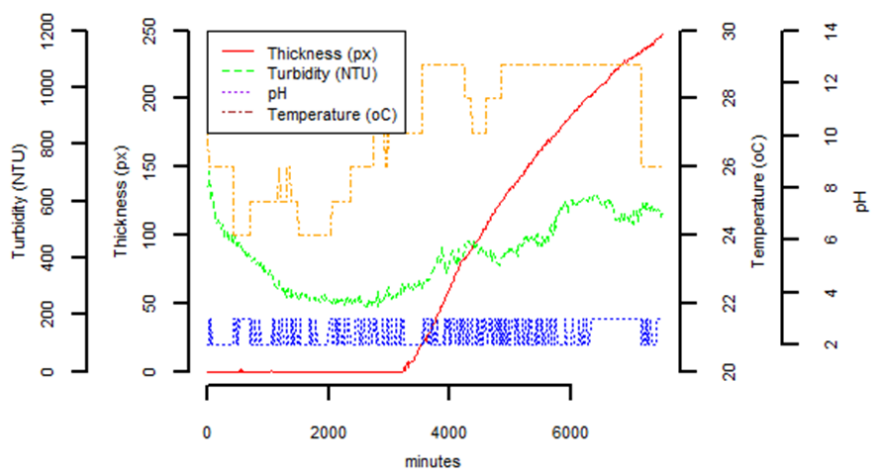

Figure 4. Thickness, turbidity, $\mathrm{pH}$, and temperature during $\mathrm{BC}$ fermentation.

Turbidity data in Figure 4 shows a different pattern. At the time of the initial inoculation, it shows a high number of turbidities, because, at the time of inoculation, the biomass that initially settles is shaken out before being poured. This shaking makes biomass is evenly distributed in the inoculum and when it is poured it will give a turbidity number to the medium in the fermenter. As shown in Figure 4, the biomass will begin to settle, which is indicated by a decrease in the turbidity number from 1000 to 3000 mins. However, along with the increase in thickness of the $\mathrm{BC}$ sheet, the turbidity rate increases, because the $\mathrm{BC}$ fibre produced by the inoculum begins to be secreted a lot into the medium before forming the $\mathrm{BC}$ sheet and influences increasing turbidity number.

Measurement of temperature in the medium shows a similar pattern with turbidity (Figure 4). At the beginning of fermentation, the temperature in the fermenter ranges from $24-26^{\circ} \mathrm{C}$. While $\mathrm{BC}$ began to visually form, there are an increase in temperature ranging from $26-29^{\circ} \mathrm{C}$. This is due to an increase in the metabolic rate of the inoculum when $\mathrm{BC}$ formed is accompanied by heat release which affects the temperature in the medium. Meanwhile, the $\mathrm{pH}$ reading data did not show any significant difference during the fermentation process.

During the fermentation process, the four sensors provide reading data and then analyse the relationship between time, thickness, turbidity, $\mathrm{pH}$, and temperature variables. The relationship between each variable is shown in Figure 5. The relationship with the highest percentage is shown between time and thickness of $94 \%$, followed by the relationship between thickness and turbidity by $80 \%$. Figure 5 shows that the fermentation time is strongly related to the thickness of the $\mathrm{BC}$ sheet formed. This can be explained that the longer the fermentation time will provide an opportunity for $G$. xylinum to secrete more $\mathrm{BC}$ fibre so that the $\mathrm{BC}$ sheet is formed. The more $\mathrm{BC}$ fibre formed, the more $\mathrm{BC}$ sheet thickness is added as shown in relation value of $80 \%$ between thickness and turbidity.

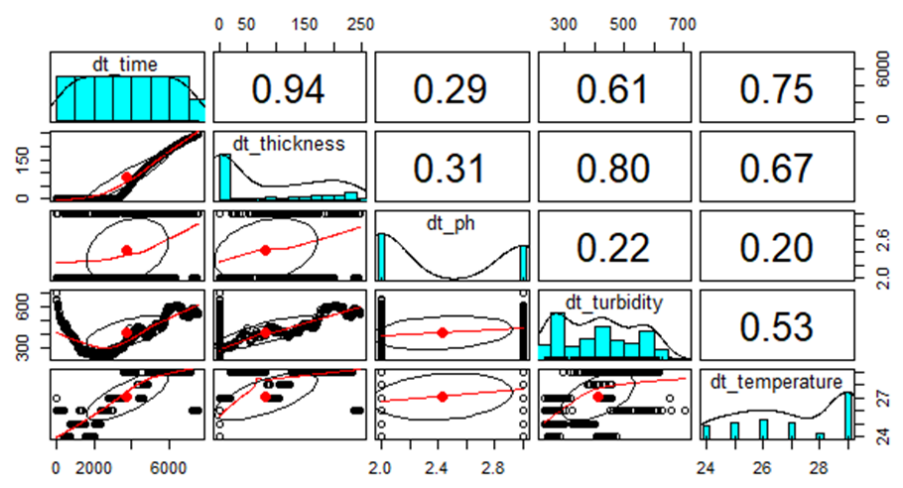

Figure 5. Variable relations of time, thickness, $\mathrm{pH}$, turbidity, temperature during $\mathrm{BC}$ fermentation 
PCA Analysis is used to reduce the dimensions of the variables used in this study. The PCA Analysis calculation uses the "prcomp" command by standardizing the data for each variable by entering the TRUE argument in the scale parameter. The results obtained 4 Principal Components in the form of PC1, $\mathrm{PC} 2, \mathrm{PC} 3$ and PC4 (Figure 6). From the figure, $\mathrm{PC} 1$ and PC2 occupy a cumulative proportion of $91.92 \%$, which means that PC1 and PC2 can capture $91.92 \%$ of the diversity of data.

$>$ summary (dataplot.pca)

Importance of components:

$\begin{array}{rrrr}\text { PC1 } & \text { PC2 } & \text { PC3 } & \text { PC4 }\end{array}$

$\begin{array}{llllllll}\text { Standard deviation } \quad & 1.7787 & 0.7123 & 0.55984 & 0.12458\end{array}$

Proportion of Variance $0.79090 .1268 \quad 0.078360 .00388$

$\begin{array}{llllll}\text { Cumulative proportion } & 0.7909 & 0.9178 & 0.99612 & 1.00000\end{array}$

$>$ dataplot.pca

standard deviations $(1, \ldots, p=4)$ :

[1] $1.77866450 .71232890 .5598402 \quad 0.1245760$

Rotation $(n \times k)=(4 \times 4)$ :

$$
\begin{array}{lrrrr} 
& \text { PC1 } & \text { PC2 } & \text { PC3 } & \text { PC4 } \\
\text { dt_time } & 0.5261107 & -0.2228180 & 0.5456057 & -0.6130857 \\
\text { dt_thickness } & 0.5427452 & 0.1799874 & 0.3718613 & 0.7312669 \\
\text { dt_turbidity } & 0.4646010 & 0.6958980 & -0.4732981 & -0.2754283 \\
\text { dt_temperature } & 0.4612819 & -0.6585458 & -0.5831145 & 0.1162491
\end{array}
$$

Figure 6 . The proportion of each principal components

Based on the relationship between time and thickness of BC in Figure 5, the data group was analysed during the fermentation process. Cluster analysis is used to estimate the number of data groupings based on the relationship between time and thickness of $\mathrm{BC}$ data. The step taken is to find the optimum cluster on the existing data relationship, using the "wss" and "silhouette" methods. The graph plot of the two methods is shown in Figure 7. The optimum number of clusters obtained between those two methods is 2 . This means that during the fermentation process there are 2 data groupings based on time and thickness data.

(a)

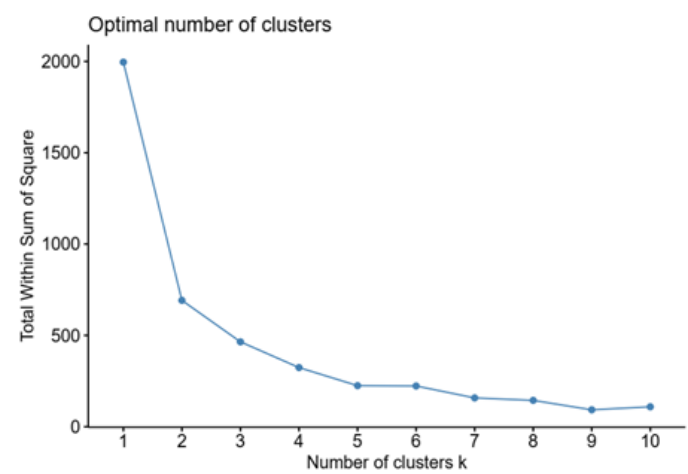

(b)

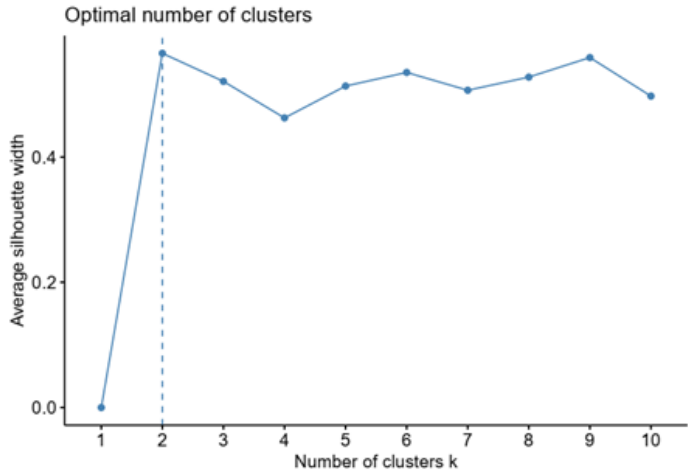

Figure 7. Optimum number of clusters obtained by (a) wss method and (b) silhouette method.
The results of the cluster analysis are used to identify the different phases in the $\mathrm{BC}$ sheet formation process. Each cluster is given a different colour attribute based on the cluster on the $\mathrm{BC}$ sheet thickness measurement graph as shown in Figure 8.

From Figure 8, during the fermentation process, there are two groups of process stages. The first cluster occurs from the stage from fermentation to the initial reading of the $\mathrm{BC}$ thickness data. This stage ends at the 61 st hour and continues to the second cluster, namely the stage of the $\mathrm{BC}$ thickness formation process. This shows that up to the 61 st hour the fermenter condition is still in the preparation phase leading to the formation of $\mathrm{BC}$ thickness, then after 61 hours, there is an increase in the formation rate of $\mathrm{BC}$ which is very different from the previous group. As shown in Figure 8, there is a sharp increase in thickness.

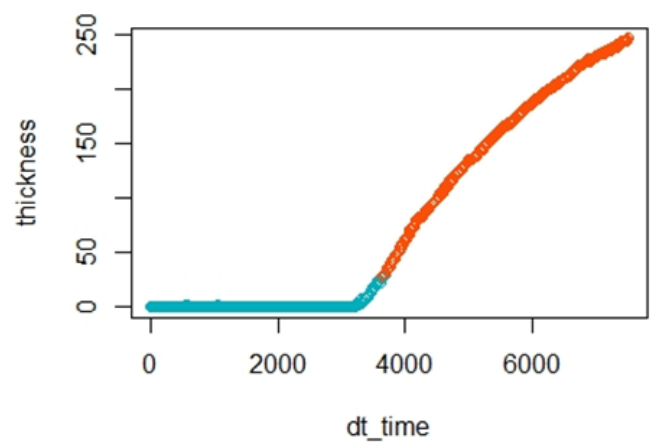

Figure 8. Graph of BC sheet thickness measurement based on clustering data during fermentation.

After obtaining a graph of the increase in thickness of $\mathrm{BC}$ sheet per unit time, an equation model that describes the speed of formation of $\mathrm{BC}$ sheet is determined. The choice of equation model is based on the largest $\mathrm{R}^{2}$ value. The Gompertz curve model using start iterations values of $a=100, b=2$ and $c=0.05$ gives $\mathrm{R}^{2}$ values 0.9972 is shown in Figure 9.

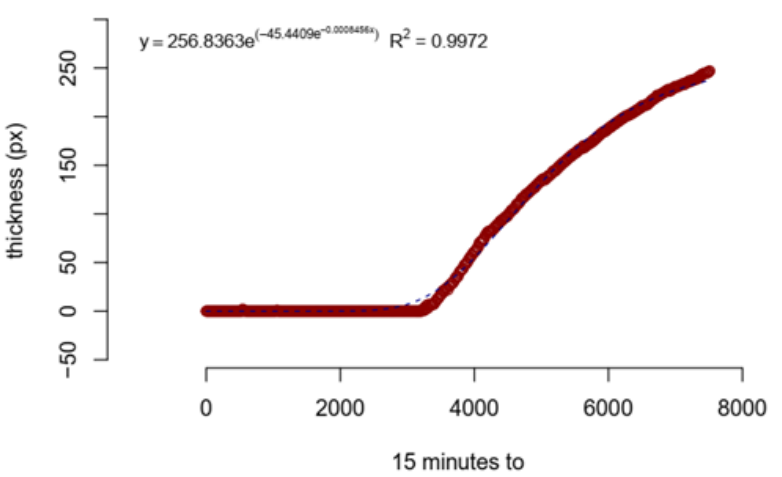

Figure 9. The plot of Gompertz curve model for BC thickness measurement during fermentation

The accuracy of the model is determined by the R2 value on the relationship between thickness and prediction of thickness based on the Gompertz model. These results are shown in Figure 10. Figure 10 shows that thickness data and thickness prediction data has a 
value of $\mathrm{R}^{2}=0.9973$. From this $\mathrm{R} 2$ showed, conclude that the Gompertz curve model is right to describe the rate of formation of $\mathrm{BC}$ sheet thickness during the fermentation process.

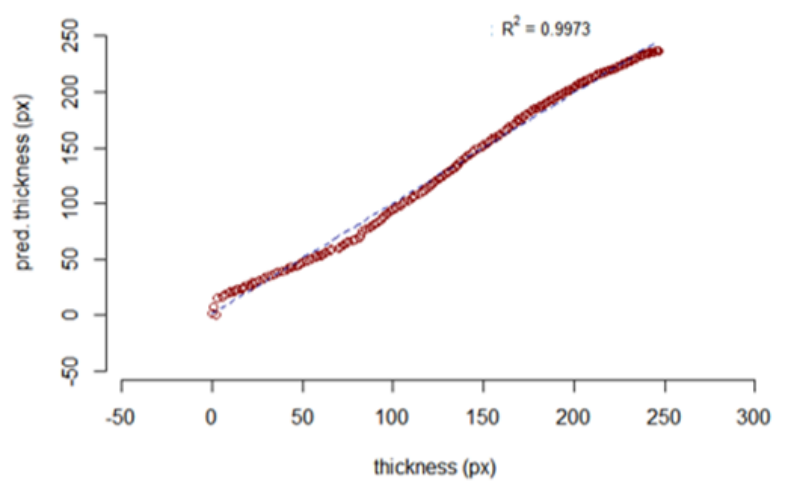

Figure 10. Relation between actual thickness and prediction of thickness during $\mathrm{BC}$ fermentation based on Gompertz model.

\section{Conclusion}

The highest correlation value was obtained from the relationship between time and thickness. The rate of $\mathrm{BC}$ formation during the fermentation process best follows the Gompertz model, with an increase in the rate occurring after the 61 st hour.

\section{Conflict of interest}

In this research and publication, there is no conflict of interest from all parties.

\section{Acknowledgments}

The authors express their gratitude to LPDP (Indonesia Endowment Fund for Education), the Ministry of Finance, and the Republic of Indonesia for providing financial support for this research.

\section{References}

Bilgi, E., Bayir, E., Sendemir-Urkmez, A. and Hames, E.E. (2016). Optimization of bacterial cellulose production by Gluconacetobacter xylinus using carob and haricot bean. International Journal of Biological Macromolecules, 90, 2-10. https:// doi.org/10.1016/j.ijbiomac.2016.02.052

Chau, C.-F., Yang, P., Yu, C.-M. and Yen, G.-C. (2008). Investigation on the Lipid- and CholesterolLowering Abilities of Biocellulose. Journal of Agricultural and Food Chemistry, 56(6), 2291-2295. https://doi.org/10.1021/jf7035802

Chawla, P.R., Bajaj, I.B., Survase, S.A. and Singhal, R.S. (2009). Microbial cellulose: Fermentative production and applications (Review). Food Technology and Biotechnology, 47(2), 107-124.
D'Ausilio, A. (2012). Arduino: A low-cost multipurpose lab equipment. Behavior Research Methods, 44(2), 305-313. https://doi.org/10.3758/s13428-011-0163-z

Hsieh, J.T., Wang, M.J., Lai, J.T. and Liu, H.S. (2016). A novel static cultivation of bacterial cellulose production by intermittent feeding strategy. Journal of the Taiwan Institute of Chemical Engineers, 63, 46-51. https://doi.org/10.1016/j.jtice.2016.03.020

Iguchi, M., Yamanaka, S. and Budhiono, A. (2000). Bacterial cellulose - a masterpiece of nature's arts. Journal of Materials Science, 35(2), 261-270. https://doi.org/10.1023/A:1004775229149

Lee, Y.-J., An, S.-J., Bae, E.-B., Gwon, H.-J., Park, J.-S., Jeong, S. I., Jeon, T.-C., Lee, H.-S., Lim, Y.-M. and Huh, J.-B. (2017). The Effect of Thickness of Resorbable Bacterial Cellulose Membrane on Guided Bone Regeneration. Materials, 10(3), 320. https://doi.org/10.3390/ma10030320

Maver, T., Maver, U., Mostegel, F., Griesser, T., Spirk, S., Smrke, D.M. and Stana-Kleinschek, K. (2015). Cellulose based thin films as a platform for drug release studies to mimick wound dressing materials. Cellulose, 22, 749-761. https://doi.org/10.1007/ s10570-014-0515-9

Mohammad, S.M. and Rahman, N.A. (2014). An Overview of Biocellulose Production Using Acetobacter xylinum Culture. Biological Research, 8 (6), 307-313.

Nugroho, D.A., Sutiarso, L., Rahayu, E.S. and Masithoh, R.E. (2020). Utilizing Real-Time Image Processing for Monitoring Bacterial Cellulose Formation During Fermentation. Agritech, 40(2), 118-123.

Purwadaria, T., Gunawan, L. and Gunawan, A.W. (2010). The Production of Nata Colored by Monascus purpureus J1 Pigments as Functional Food. Microbiology Indonesia, 4(1), 6-10. https:// doi.org/10.5454/mi.4.1.2

Sheykhnazari, S., Tabarsa, T., Ashori, A., Shakeri, A. and Golalipour, M. (2011). Bacterial synthesized cellulose nanofibers; Effects of growth times and culture mediums on the structural characteristics. Carbohydrate Polymers, 86(3), 1187-1191. https:// doi.org/10.1016/j.carbpol.2011.06.011

Ullah, H., Santos, H.A. and Khan, T. (2016). Applications of bacterial cellulose in food, cosmetics and drug delivery. Cellulose, 23(4), 2291-2314. https://doi.org/10.1007/s10570-016-0986-y

$\mathrm{Wu}, \mathrm{S}$. and Lia, Y. (2008). Application of bacterial cellulose pellets in enzyme immobilization. Journal of Molecular Catalysis B: Enzymatic, 54(3-4), 103108. https://doi.org/10.1016/j.molcatb.2007.12.021 